\title{
Fast kilovoltage (KV)-switching dual-energy computed tomography hydroxyapatite (HAP)-water decomposition technique for identifying bone marrow edema in vertebral compression fractures
}

\author{
Junhan Pan ${ }^{1}$, Luyou Yan ${ }^{1}$, Hui Gao ${ }^{1}$, Yewen $\mathrm{He}^{1}$, Zeya Zhong ${ }^{1}$, Ping Li $^{1}$, Yaxi Zhang ${ }^{2}$, Ying Guo ${ }^{2}$, \\ Liangying Liao', Shuwei Zhou', Kun Zhang ${ }^{1,3}$ \\ ${ }^{1}$ Department of Radiology, First Affiliated Hospital of Hunan University of Chinese Medicine, Changsha 410007, China; ${ }^{2}$ GE Healthcare (Shanghai) \\ Co., Ltd., Shanghai 201203, China; ${ }^{3}$ College of Integrated Traditional Chinese and Western Medicine, Hunan University of Chinese Medicine, \\ Changsha 410208, China
}

Correspondence to: Kun Zhang. Department of Radiology, First Affiliated Hospital of Hunan University of Chinese Medicine, 95 Shaoshan Middle Road, Yuhua District, Changsha 410007, China; College of Integrated Traditional Chinese and Western Medicine, Hunan University of Chinese Medicine, 300 Xueshi Road, Yuelu District, Changsha 410208, China. Email: kun_zhang0102@163.com.

Background: Hydroxyapatite (HAP) is the main component of bone mineral. The utility of using HAPwater decomposition technique with fast kilovoltage (KV)-switching dual-energy computed tomography (DECT) to detect abnormal edema in vertebral compression fractures (VCFs) has not been widely reported.

Methods: A total of 31 consecutive patients with 80 VCFs who underwent DECT and magnetic resonance imaging (MRI) of the spine were retrospectively enrolled in our study between October 2018 and January 2019. VCFs in MR examinations served as the standard of reference. Two radiologists blindly and independently evaluated color-coded overlay virtual nonhydroxyapatite (VNHAP) images for the presence of abnormal edema. The inter-reader agreement, specificity, sensitivity, accuracy, and predictive values of VNHAP images for edema detection were calculated. The diagnostic accuracy of two readers was compared using McNemar's test. Two additional radiologists performed a quantitative analysis on VNHAP images, receiver operating characteristic (ROC) curve analysis was conducted, and the threshold was calculated.

Results: MRI depicted 45 edematous and 35 nonedematous VCFs. For visual analysis, the VNHAP technique showed a sensitivity of $93.3 \%$, a specificity of $97.1 \%$, a positive predictive value (PPV) of $97.7 \%$, a negative predictive value (NPV) of $91.9 \%$, and an accuracy of $95.0 \%$. The inter-reader agreement was almost perfect $(\mathrm{k}=0.90)$. The diagnostic accuracy of the two readers showed no significant differences in the assessment of VNHAP images $(\mathrm{P}=1.00)$. Significant differences in CT numbers between vertebrae with and without bone marrow edema were found by quantitative analysis $(\mathrm{P}<0.01)$. The area under the curve (AUC) of the VNHAP images was estimated to be 0.917 . The threshold of $1,003.2 \mathrm{mg} / \mathrm{cm}^{3}$ yielded a sensitivity of $88.9 \%$ and a specificity of $82.9 \%$ for the differentiation of fresh and old VCFs.

Conclusions: Fast KV-switching DECT HAP-water decomposition technique had excellent diagnostic performance for identifying acute and chronic VCFs in visual and quantitative analyses.

Keywords: Dual-energy computed tomography (DECT); material decomposition; bone marrow edema; compression fracture; spine

Submitted Jul 06, 2019. Accepted for publication Feb 18, 2020.

doi: 10.21037/qims.2020.02.16

View this article at: http://dx.doi.org/10.21037/qims.2020.02.16 


\section{Introduction}

Vertebral compression fractures (VCFs) of the thoracolumbar spine are common age-related clinical issues and are associated with an increased risk of morbidity and mortality $(1,2)$. In comparison with appendicular fractures, thoracolumbar compression fractures are often asymptomatic and manifest as misleading pain complaints, resulting in underreporting by clinicians or radiologists (3). It has thus become necessary to find an accurate and effective diagnostic examination to detect and localize thoracolumbar compression fractures for the appropriate treatment and prevention of associated complications $(4,5)$.

For VCFs, imaging procedures are often used as a helpful tool for determining the relative location and treatment choice (6). Traditionally, magnetic resonance imaging (MRI) is a reliable modality to detect abnormal edema and fracture morphology in VCFs (7-9). However, due to its high cost, long examination period, and multiple contraindications, the application of MRI remains limited. Conventional computed tomography (CT) produces an exquisite depiction of subtle cortical fractures but lacks accuracy regarding the identification of acute and old fractures $(3,8)$. Recently, the technological innovation of fast $\mathrm{KV}$-switching dual-energy CT (DECT) has enabled the detection and characterization of bone marrow abnormalities, and may soon be preferentially used over conventional CT for musculoskeletal imaging.

Depending on different photoelectric effect and Compton scattering of materials at two X-ray energy levels, DECT allows for material discrimination and attenuation measurement $(6,10-12)$. DECT has the unique ability to differentiate a variety of material types based on differential $\mathrm{X}$-ray attenuation at different photon energies. Recent studies have reported that the use of DECT with the virtual noncalcium (VNCa) technique can produce high sensitivity and specificity in the detection of bone marrow edema in virtual and quantitative analyses $(6,12-14)$. Liao et al., previously demonstrated that hydroxyapatite [HAP, $\left.\mathrm{Ca}_{10}\left(\mathrm{PO}_{4}\right)_{6}(\mathrm{OH})_{2}\right]$ has a higher level of similarity to the true composition of the bone mineral than calcium (15). Nevertheless, the actual usefulness of the DECT virtual nonhydroxyapatite (VNHAP) technique for detecting abnormal edema remains unknown.

The aim of our study was to evaluate the performance of DECT HAP-water decomposition technique in the detection of the acute or chronic VCFs, using MRI as the reference standard. Our study may provide the possibility of using DECT to generate clinical and health-economic benefits.

\section{Methods}

\section{Patient selection}

This prospectively defined retrospective study was approved by the local ethics committee, and written informed consent was waived. A total of 108 consecutive patients with at least one thoracolumbar fracture who underwent both DECT and MR within 3 days between October 2018 and January 2019 were enrolled. Exclusion criteria included spinal tumor, spinal infection, surgical history for VCFs, and basic disease or drugs affecting the phosphorus and calcium metabolism. The final study group comprised 31 patients (mean age \pm standard deviation, $70.7 \pm 13.1$ years; range, 27 92 years), from whom 80 vertebral bodies were evaluated. The study population included 9 men (mean age \pm standard deviation, $62.8 \pm 18.4$ years; range, $30-81$ years) and 22 women (mean age \pm standard deviation, $74.0 \pm 8.8$ years; range, 51-90 years); further details are shown in Figure 1.

\section{Image acquisition}

A single-source DECT system (Revolution, GE Medical Systems, Milwaukee, WI, USA) was employed to obtain CT scans. The acquisition parameters were applied as follows: GSI KV mode with rapid KV switching between 80 and $140 \mathrm{kVp}$; tube current, $190 \mathrm{~mA}$; collimation thickness, $0.625 \mathrm{~mm}$; helical scan type; rotation time, $0.8 \mathrm{~s}$; helical pitch, 0.984; 50\% ASIR-V; CTDIvol, $7.37 \mathrm{mGy}$; and bone reconstruction type.

MRI was conducted on a 3.0-T system and a whole spine coil (HDxT, GE Medical Systems, Milwaukee, WI, USA). Images were obtained with the following parameters: a sagittal T1-weighted FSE sequence [repetition time (TR) $480 \mathrm{~ms}$, echo time (TE) $11 \mathrm{~ms}, 3 \mathrm{~mm}$ slice thickness, $1 \mathrm{~mm}$ gap, echo train length $(\mathrm{ETL})=5$, number of excitations $(\mathrm{NEX})=2$ ]; a sagittal T2-weighted IDEAL sequence (TR 2,320 ms, TE $85 \mathrm{~ms}, 3 \mathrm{~mm}$ slice thickness, $1 \mathrm{~mm}$ gap, ETL =15, NEX =2), in-phase images, water images, and fat images being automatically calculated; and axial T2-weighted FSE sequence (TR 3,200 ms, TE $120 \mathrm{~ms}$, $3.5 \mathrm{~mm}$ slice thickness, $1 \mathrm{~mm}$ gap, $\mathrm{ETL}=25, \mathrm{NEX}=2$ ).

\section{Image postprocessing}

The data from DECT images were postprocessed using an advanced workstation (ADW4.6; GE Medical Systems, Milwaukee, WI, USA). The VNHAP images were generated by subtracting HAP, and the virtual HAP images were 


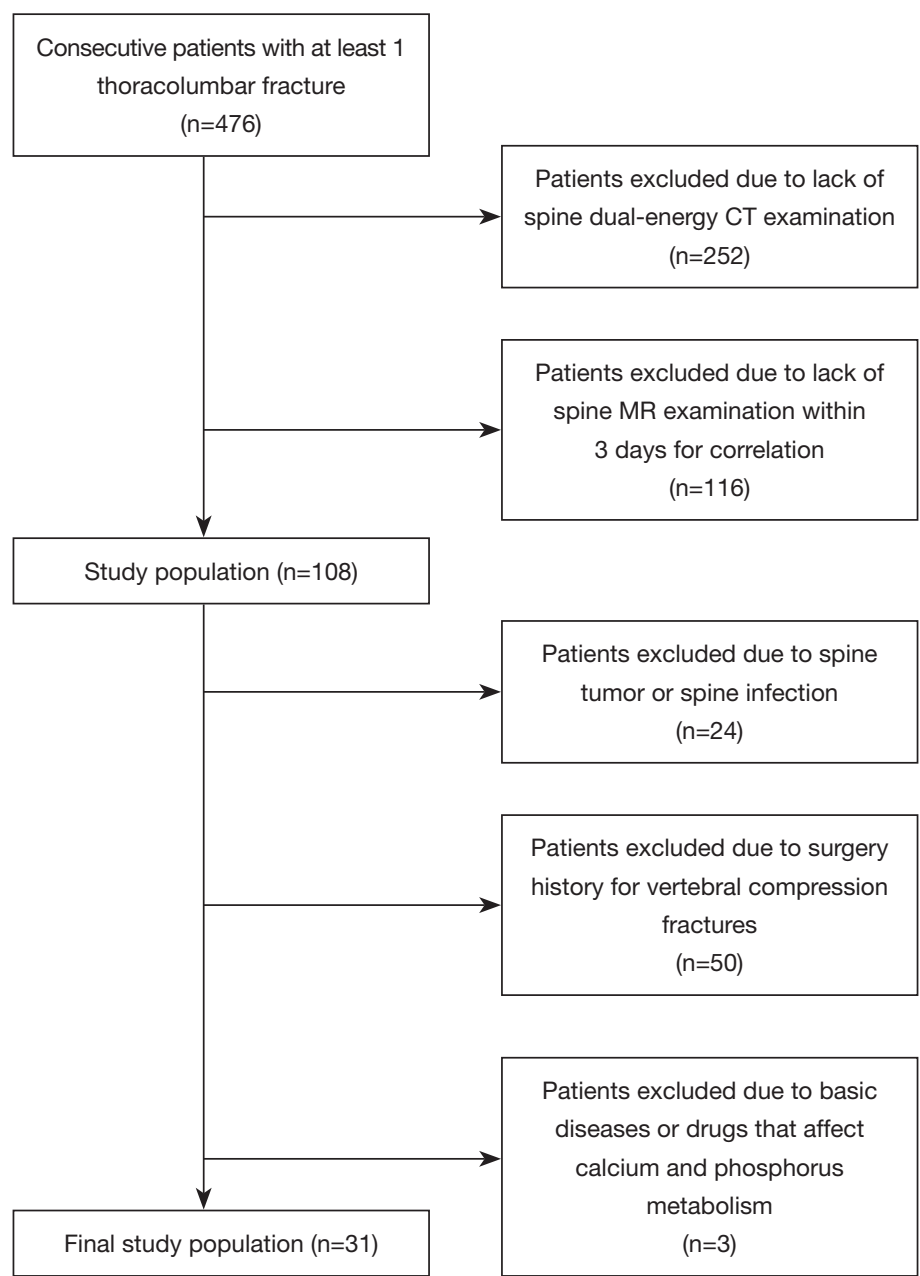

Figure 1 Flowchart showing inclusion and exclusion criteria. CT, computed tomography.

generated by subtracting water. For further analysis, colorcoded overlay VNHAP images were generated by merging the VNHAP images with $70 \mathrm{keV}$ monoenergetic CT images.

\section{Image analysis}

MR images of fractured vertebrae were individually displayed in a randomized fashion and visually analyzed by a radiologist (K Zhang, with 13 years of experience in musculoskeletal radiology). This reader blindly and independently evaluated each vertebra for the presence of abnormal edema and determined the age of the fractures using a binary classification $(0=$ chronic compression fracture; 1 = acute compression fracture). Accordingly, two additional radiologists ( $\mathrm{L}$ Yan reader 1, and $\mathrm{H}$ Gao reader 2, with 5 and 10 years of experience, respectively), without the knowledge of the MR results, independently evaluated the color-coded overlay VNHAP images in a random order. In instances of inter-reader disagreement, a consensus reading was appended. Consensual results were used to determine the presence of abnormal edema and for further analysis. Each observer was free to adjust window settings and the magnification factor of the images.

Quantitative images analysis was performed after the application of a visual analysis by a senior radiologist (Y He, with 8 years of experience) and a radiology resident (J Pan, with 2 years of experience) together on the sagittal plane. According to the position of the highest signal on MR T2weighted images (T2WI), two consensus-derived regions of interest (ROIs) varying from 25 to $100 \mathrm{~mm}^{2}$ were placed on the anterior and posterior part of the vertebrae, respectively. Our ROIs were placed at least $2 \mathrm{~mm}$ away from the cortical bone, and excluded central veins or bone islands. The average CT numbers of two measurements were calculated 

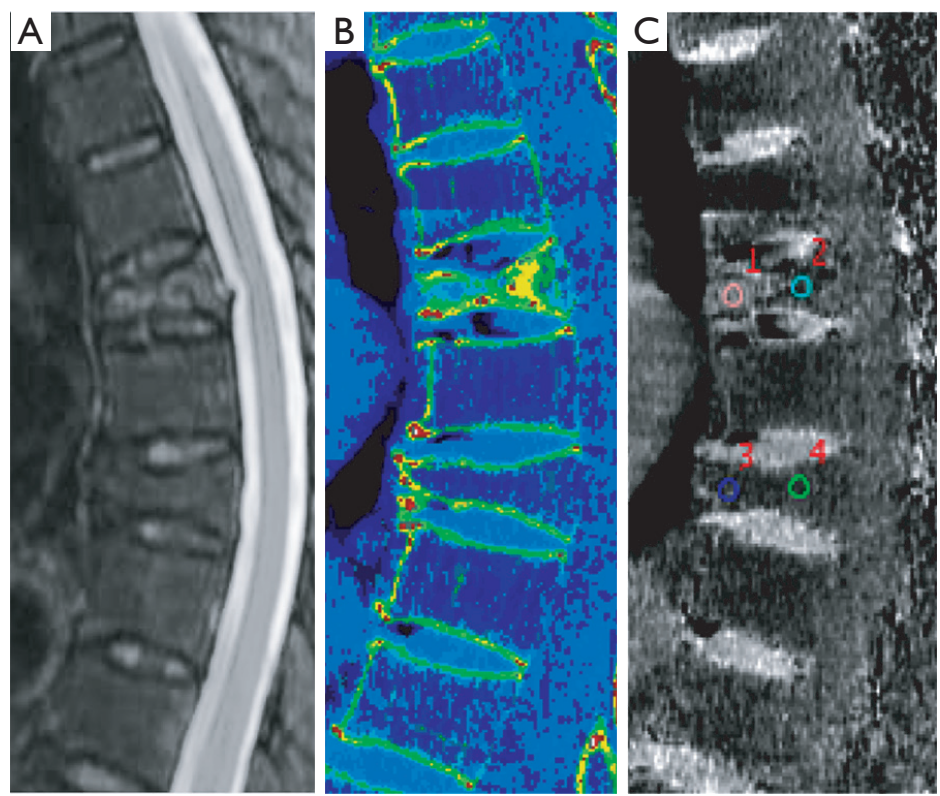

Figure 2 Images from a 68-year-old man with compression fractures at T7 and T9. (A) Sagittal spin-echo T2-weighted MR image confirmed abnormal edema in the T7 body, which is coded green in (B), whereas the collapsed nonedematous T9 body was of chronic nature. (B) Color-coded virtual nonhydroxyapatite (VNHAP) image revealed bone marrow edema of the T7 body. (C) Sagittal VNHAP grayscale image with ROIs. The mean CT numbers of ROIs across the T7 body (mean 1,012.7 $\mathrm{mg} / \mathrm{cm}^{3}$ ) were higher than that of the T9 body (mean $993.3 \mathrm{mg} / \mathrm{cm}^{3}$ ). MR, magnetic resonance; CT, computed tomography; ROIs, regions of interest.

for subsequent analysis.

\section{Statistical analysis}

Continuous variables are expressed as mean \pm standard deviation, and MRI served as the reference standard. For visual analysis, the sensitivity, specificity, accuracy, and predictive values were calculated using a constructed contingency table. The inter-reader agreement was measured with k-statistic (16). McNemar's test was used to compare the diagnostic accuracy of the two readers in evaluating VNHAP images. For quantitative analysis, the data was tested for homogeneity and normality using the Levene's and Kolmogorov-Smirnov tests, respectively. In addition, we performed the Student's $t$-test for comparing CT numbers obtained from VNHAP images between the acute and chronic VCF groups. A receiver operating characteristic (ROC) curve was constructed for the determination of the threshold that best separated the two classifications. The area under the curve (AUC), with corresponding sensitivity and specificity, was calculated. SPSS statistical software (v.20.0; IBM) was used for all analyses. $\mathrm{P}<0.05$ was considered to indicate a statistically significant difference.

\section{Results}

In our study group, a total of 80 vertebrae were identified in 31 patients. According to MR findings, 45 fractures (56.3\%) and 35 fractures (43.8\%) were categorized as acute and chronic compression fractures, respectively. In addition, there were $34(42.5 \%)$ thoracic and $46(57.5 \%)$ lumbar vertebrae (Figure 2).

\section{Visual image analysis}

Diagnostic performance of the visual assessment is presented in Table 1. A consensus for edema assessment on color-coded overlay VNHAP images was required in five vertebral bodies. Among all depicted findings, there were three false negative cases and one false positive case. Interreader agreement was excellent in visual analysis $(\mathrm{k}=0.90)$. No significant differences were observed in the diagnostic accuracy between the two readers $(\mathrm{P}=1.00)$. 
Table 1 Diagnostic performance of dual-energy CT in the visual evaluation of bone marrow edema in vertebral compression fractures

\begin{tabular}{lccc}
\hline Variables & Reader 1 & Reader 2 & Consensus results \\
\hline Sensitivity & $41 / 45,91.1 \%(78.8 \%, 97.5 \%)$ & $40 / 45,88.9 \%(75.9 \%, 96.3 \%)$ & $42 / 45,93.3 \%(81.7 \%, 98.6 \%)$ \\
Specificity & $34 / 35,97.1 \%(85.1 \%, 99.9 \%)$ & $33 / 35,94.3 \%(80.8 \%, 99.3 \%)$ & $34 / 35,97.1 \%(85.1 \%, 99.9 \%)$ \\
PPV & $41 / 42,97.6 \%(85.6 \%, 99.6 \%)$ & $40 / 42,95.2 \%(83.8 \%, 98.7 \%)$ & $42 / 43,97.7 \%(85.9 \%, 99.7 \%)$ \\
NPV & $34 / 38,89.5 \%(76.9 \%, 95.6 \%)$ & $33 / 38,86.8 \%(74.2 \%, 93.8 \%)$ & $34 / 37,91.9 \%(79.1 \%, 97.1 \%)$ \\
Accuracy & $75 / 80,93.8 \%(86.2 \%, 97.3 \%)$ & $73 / 80,91.3 \%(83.0 \%, 95.7 \%)$ & $76 / 80,95.0 \%(87.8 \%, 98.0 \%)$ \\
\hline
\end{tabular}

Numbers in parentheses are 95\% confidence interval. CT, computed tomography; PPV, positive predictive value; NPV, negative predictive value.

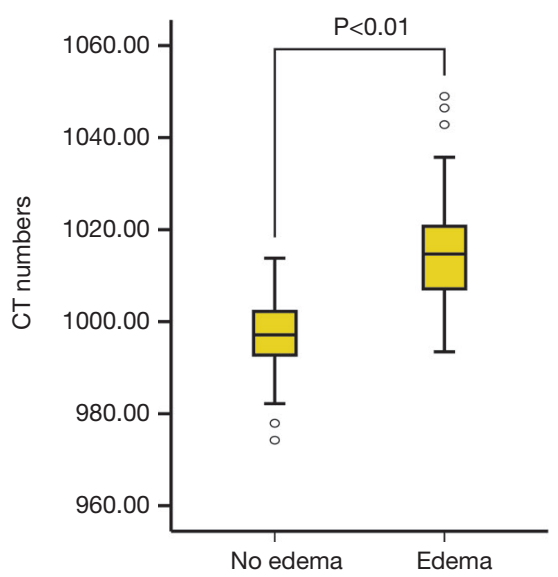

Figure 3 Comparison of CT numbers on virtual nonhydroxyapatite (VNHAP) images between edematous vertebrae and nonedematous vertebrae. CT numbers in edematous vertebral bodies (mean $1,015.8 \pm 11.9 \mathrm{mg} / \mathrm{cm}^{3}$ ) were significantly higher than those in nonedematous vertebral bodies (mean $997.0 \pm 9.3 \mathrm{mg} / \mathrm{cm}^{3}$ ) $(\mathrm{P}<0.01)$. The data were tested for homogeneity and normality using the Levene's and Kolmogorov-Smirnov test, respectively (all $\mathrm{P}>0.05)$. CT, computed tomography.

\section{Quantitative VNHAP image evaluation}

Mean CT numbers on VNHAP images were $1,015.8 \pm 11$.9 and $997.0 \pm 9.3 \mathrm{mg} / \mathrm{cm}^{3}$ for edematous and nonedematous vertebral bodies, respectively. There was a significant difference between the groups ( $t=7.7, \mathrm{P}<0.01$, Figure 3$)$. The ROC curve analysis achieved an AUC of 0.917, and the optimal threshold of $1,003.2 \mathrm{mg} / \mathrm{cm}^{3}$ provided a sensitivity of $88.9 \%$ (95\% CI: $75.9 \%, 96.3 \%)$ and a specificity of 82.9\% (95\% CI: 66.4\%, 93.4\%) (Figures 4).

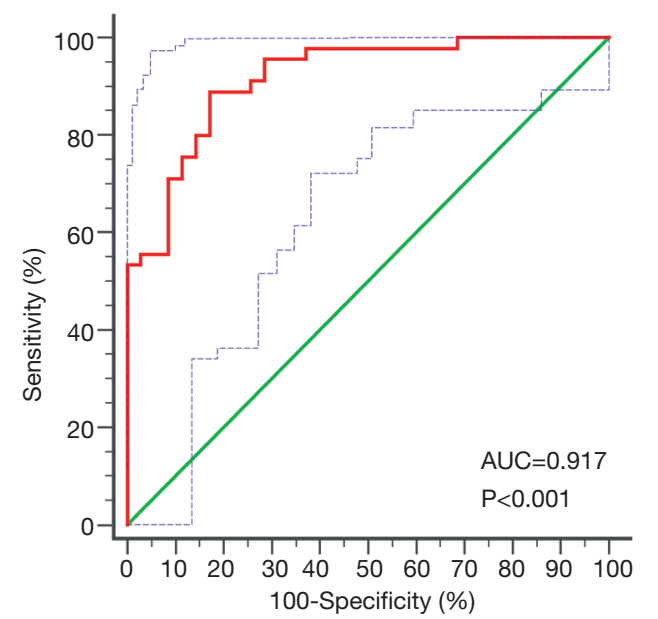

Figure 4 ROC curve of quantitative evaluation. Area under the curve (solid red line) was 0.917 (95\% CI: 0.834, 0.967) (dotted blue line), which indicated good diagnostic ability. AUC, area under the curve; ROC, receiver operating characteristic; CI, confidence interval.

\section{Discussion}

Our study demonstrated that the HAP-water decomposition technique of fast $\mathrm{KV}$-switching DECT represents a feasible tool to identify and characterize abnormal edema in VCFs. Detected abnormal edema on VNHAP images was revealed by subtracting HAP signal from trabecular bone. In our study, we were able to accomplish these results with high sensitivity, specificity, and accuracy.

The fast KV-switching DECT system, which employs a single $\mathrm{x}$-ray source that rapidly changes energy settings and collects data at high and low energy scans in every single rotation, has been recently implemented in clinical 
practice $(14,17)$. Several studies have proven the ability of the DECT VNCa technique for detecting abnormal edema in limbs $(11,14,18,19)$ and the spine $(6,12,13,20-22)$. While it is known that bones are composed of HAP crystals embedded in a collagenous matrix, the use of VNHAP protocol in the identification of fresh VCFs has not been fully clarified.

The performance of two readers in visual analysis demonstrated a slight difference between reader 1 and reader 2 who had different experience levels (sensitivity of $91.1 \%$ vs. $88.9 \%$ and a specificity of $97.1 \%$ vs. $94.3 \%$, for the more experienced and less experienced reader, respectively). However, no statistically significant differences were observed between the diagnostic accuracy of the two readers $(\mathrm{P}=1.00)$, and the inter-reader agreement was excellent $(\mathrm{k}=0.90)$, indicating that the VNHAP algorithm could be a promising technique in clinical workflow. This result is in agreement with previously published research that found qualitative analysis findings depend on multiple technical factors and the personal routine of the readers $(13,23)$. In spite of this, some differences between the readers did exist in our results. Notably, one false positive finding and three false negative findings were observed in visual image interpretation. The false positive finding may be associated with the region of increased red marrow in the vertebral body (21). Despite there being room for further technological development, we have to acknowledge that abnormal attenuation of edema in VNHAP protocol can be imitated by the mixture of fat and red marrow (11). Conversely, the false negative findings often occurred in subtle fractures located adjacent to cortical bone, resulting from spatial averaging effects. The previous study showed the outperformance of MRI for detecting fractures beneath the cortex, which was further confirmed by the result from our study. Another possible reason for the false negative findings was bone sclerosis. In this case, the sclerosis might have caused an abnormal attenuation, masking the edema on VNHAP images, and thus led to underreporting. Furthermore, a high negative predictive value of $91.9 \%$ in visual analysis substantiated the feasibility of DECT VNHAP protocol for excluding acute fractures.

The underlying principle of determining the threshold for bone marrow edema can be explained by the fact that the fluid (relatively high-attenuating) may substitute for the yellow marrow (a relatively low-attenuating substance) resulting in increased CT numbers in measurement (24). The optimal threshold of $1,003.2 \mathrm{mg} / \mathrm{cm}^{3}$ found in our study was significantly higher than previous findings in
VNCa images $(6,12,21)$. The difference may stem from the use of different material decomposition techniques, scanner hardware, and KV settings. Meanwhile, high sensitivity and specificity $(88.9 \%$ vs. $82.9 \%)$ of the optimal threshold in detecting abnormal edema validated that DECT VNHAP quantitative analysis could be a promising technique for the diagnosis and identification of chronic and acute vertebral fractures.

Interestingly, visual analysis exhibited a higher sensitivity (93.3\%) and specificity (97.1\%) than ROI-based density measurements analysis (sensitivity, $88.9 \%$; specificity, $82.9 \%$ ) in our study. The result was in accordance with a meta-analysis which reported the superiority of visual evaluation in differentiating fresh from old fractures (25). Therefore, it suggests that visual VNHAP image analysis could serve as a viable screening tool besides MRI for diagnosing VCFs in future clinical practice.

Some limitations of this study should be discussed. Firstly, the number of patient samples was relatively small. This is due to the fact that only patients who had undergone a DECT study within 3 days of the MR examination were selected for our study. Moreover, there were more fresh fractures included in our study cohort, which was probably a result of only subjects with a clinically suspected fresh fracture being admitted to CT examination. At last, we excluded the patients with diagnosed spinal infections, spinal tumors, and diseases affecting calcium metabolism. The abnormal marrow composition changes in these subjects could be discussed in further investigation.

To conclude, our study demonstrated the feasibility of using DECT VNHAP images to depict abnormal edema in VCFs, at both qualitative and quantitative analysis. On the basis of this initial experience with DECT HAPwater decomposition technique in diagnosing vertebral fractures, further studies should focus on the comparison of diagnostic accuracy between different basic material pairs in order to determine whether patients need an additional MR examination.

The sample size consideration is shown in the supplementary material.

\section{Acknowledgments}

Funding: None.

\section{Footnote}

Conflicts of Interest: The authors have no conflicts of interest 
to declare.

Ethical Statement: This prospectively defined retrospective study was approved by the local ethics committee, and written informed consent was waived.

Open Access Statement: This is an Open Access article distributed in accordance with the Creative Commons Attribution-NonCommercial-NoDerivs 4.0 International License (CC BY-NC-ND 4.0), which permits the noncommercial replication and distribution of the article with the strict proviso that no changes or edits are made and the original work is properly cited (including links to both the formal publication through the relevant DOI and the license). See: https://creativecommons.org/licenses/by-nc-nd/4.0/.

\section{References}

1. Center JR, Nguyen TV, Schneider D, Sambrook PN, Eisman JA. Mortality after all major types of osteoporotic fracture in men and women: an observational study. Lancet 1999;353:878-82.

2. Lindsay R, Silverman SL, Cooper C, Hanley DA, Barton I, Broy SB, Licata A, Benhamou L, Geusens P, Flowers K, Stracke H, Seeman E. Risk of new vertebral fracture in the year following a fracture. JAMA 2001;285:320-3.

3. Carberry GA, Pooler BD, Binkley N, Lauder TB, Bruce RJ, Pickhardt PJ. Unreported vertebral body compression fractures at abdominal multidetector CT. Radiology 2013;268:120-6.

4. Klazen CA, Lohle PN, de Vries J, Jansen FH, Tielbeek AV, Blonk MC, Venmans A, van Rooij WJ, Schoemaker MC, Juttmann JR, Lo TH, Verhaar HJ, van der Graaf Y, van Everdingen KJ, Muller AF, Elgersma OE, Halkema DR, Fransen H, Janssens X, Buskens E, Mali WP. Vertebroplasty versus conservative treatment in acute osteoporotic vertebral compression fractures (Vertos II): an open-label randomised trial. Lancet 2010;376:1085-92.

5. Delmas PD. Treatment of postmenopausal osteoporosis. Lancet 2002;359:2018-26.

6. Wang CK, Tsai JM, Chuang MT, Wang MT, Huang KY, Lin RM. Bone marrow edema in vertebral compression fractures: detection with dual-energy CT. Radiology 2013;269:525-33.

7. Piazzolla A, Solarino G, Lamartina C, De Giorgi S, Bizzoca D, Berjano P, Garofalo N, Setti S, Dicuonzo F, Moretti B. Vertebral Bone Marrow Edema (VBME) in Conservatively Treated Acute Vertebral Compression
Fractures (VCFs): Evolution and Clinical Correlations. Spine (Phila Pa 1976) 2015;40:E842-8.

8. Memarsadeghi M, Breitenseher MJ, Schaefer-Prokop C, Weber M, Aldrian S, Gabler C, Prokop M. Occult scaphoid fractures: comparison of multidetector $\mathrm{CT}$ and MR imaging--initial experience. Radiology 2006;240:169-76.

9. Wáng YXJ, Che-Nordin N. Some radiographically 'occult' osteoporotic vertebral fractures can be evidential if we look carefully. Quant Imaging Med Surg 2019;9:1992-5.

10. McCollough CH, Leng S, Yu L, Fletcher JG. Dual- and Multi-Energy CT: Principles, Technical Approaches, and Clinical Applications. Radiology 2015;276:637-53.

11. Pache G, Krauss B, Strohm P, Saueressig U, Blanke P, Bulla S, Schafer O, Helwig P, Kotter E, Langer M, Baumann T. Dual-energy CT virtual noncalcium technique: detecting posttraumatic bone marrow lesions-feasibility study. Radiology 2010;256:617-24.

12. Petritsch B, Kosmala A, Weng AM, Krauss B, Heidemeier A, Wagner R, Heintel TM, Gassenmaier T, Bley TA. Vertebral Compression Fractures: ThirdGeneration Dual-Energy CT for Detection of Bone Marrow Edema at Visual and Quantitative Analyses. Radiology 2017;284:161-8.

13. Kaup M, Wichmann JL, Scholtz JE, Beeres M, Kromen W, Albrecht MH, Lehnert T, Boettcher M, Vogl TJ, Bauer RW. Dual-Energy CT-based Display of Bone Marrow Edema in Osteoporotic Vertebral Compression Fractures: Impact on Diagnostic Accuracy of Radiologists with Varying Levels of Experience in Correlation to MR Imaging. Radiology 2016;280:510-9.

14. Mallinson PI, Coupal TM, McLaughlin PD, Nicolaou S, Munk PL, Ouellette HA. Dual-Energy CT for the Musculoskeletal System. Radiology 2016;281:690-707.

15. Liao EY, Wu XP, Luo XH, Zhang H, Dai RC, Huang G, Wang WB. Establishment and evaluation of bone mineral density reference databases appropriate for diagnosis and evaluation of osteoporosis in Chinese women. J Bone Miner Metab 2003;21:184-92.

16. Landis JR, Koch GG. The measurement of observer agreement for categorical data. Biometrics 1977;33:159-74.

17. Xie T, Li Y, He G, Zhang Z, Shi Q, Cheng G. The influence of liver fat deposition on the quantification of the liver-iron fraction using fast-kilovolt-peak switching dual-energy CT imaging and material decomposition technique: an in vitro experimental study. Quant Imaging Med Surg 2019;9:654-61.

18. Guggenberger R, Gnannt R, Hodler J, Krauss B, Wanner 
GA, Csuka E, Payne B, Frauenfelder T, Andreisek G, Alkadhi H. Diagnostic performance of dual-energy CT for the detection of traumatic bone marrow lesions in the ankle: comparison with MR imaging. Radiology 2012;264:164-73.

19. Karaca L, Yuceler Z, Kantarci M, Cakir M, Sade R, Calikoglu C, Ogul H, Bayrakturan UG. The feasibility of dual-energy CT in differentiation of vertebral compression fractures. Br J Radiol 2016;89:20150300.

20. Akisato K, Nishihara R, Okazaki H, Masuda T, Hironobe A, Ishizaki H, Shota K, Yamaguchi H, Funama Y. Dual-Energy CT of Material Decomposition Analysis for Detection with Bone Marrow Edema in Patients with Vertebral Compression Fractures. Acad Radiol 2020;27:227-32.

21. Bierry G, Venkatasamy A, Kremer S, Dosch JC, Dietemann JL. Dual-energy CT in vertebral compression

Cite this article as: Pan J, Yan L, Gao H, He Y, Zhong Z, Li P, Zhang Y, Guo Y, Liao L, Zhou S, Zhang K. Fast kilovoltage (KV)-switching dual-energy computed tomography hydroxyapatite (HAP)-water decomposition technique for identifying bone marrow edema in vertebral compression fractures. Quant Imaging Med Surg 2020;10(3):604-611. doi: 10.21037/qims.2020.02.16 fractures: performance of visual and quantitative analysis for bone marrow edema demonstration with comparison to MRI. Skeletal Radiol 2014;43:485-92.

22. Kosmala A, Weng AM, Heidemeier A, Krauss B, Knop S, Bley TA, Petritsch B. Multiple Myeloma and DualEnergy CT: Diagnostic Accuracy of Virtual Noncalcium Technique for Detection of Bone Marrow Infiltration of the Spine and Pelvis. Radiology 2018;286:205-13.

23. Griffith JF. Identifying osteoporotic vertebral fracture. Quant Imaging Med Surg 2015;5:592-602.

24. Mandalia V, Henson JH. Traumatic bone bruising--a review article. Eur J Radiol 2008;67:54-61.

25. Suh CH, Yun SJ, Jin W, Lee SH, Park SY, Ryu CW. Diagnostic performance of dual-energy CT for the detection of bone marrow oedema: a systematic review and meta-analysis. Eur Radiol 2018;28:4182-94. 


\section{Supplementary}

\section{Sample size estimation}

The sample size of the diagnostic test was calculated as follows:

$$
\begin{aligned}
& n_{1}=\frac{Z_{\mathrm{a}}^{2} \operatorname{Sen}(1-\operatorname{Sen})}{\delta^{2}} \\
& n_{2}=\frac{Z_{\mathrm{a}}^{2} \operatorname{Spe}(1-\operatorname{Spe})}{\delta^{2}}
\end{aligned}
$$

Note: $n 1=$ Vertebra number with acute VCF; $n 2=$ Vertebra number with chronic VCF; $\alpha=0.05, Z_{\alpha}=1.96$, $\delta=0.10$, Sen $=$ Sensitivity, Spe $=$ Specificity .

In previous studies $(6,13,22)$, dual-energy computed tomography provided a sensitivity of $90-96 \%$ and a specificity of $90-98 \%$ for the detection of abnormal bone marrow, so the sensitivity and specificity were both set at 0.9 in our study, and the sample size was about 33 per group (the acute compression fractures group and the chronic compression fractures group, respectively). The sample sizes of our study were more than the minimum required sample sizes. 